EESTI NSV TEADUSTE AKADEEMIA TOIMETISED. XII KÖIDE

FUUSIKA-MATEMAATIKA- JA TEHNIKATEADUSTE SEERIA. 1963, NR. 2

ИЗВЕСТИЯ АКАДЕМИИ НАУК ЭСТОНСКОЙ ССР. ТОМ ХІІ СЕРИЯ ФИЗИКО-МАТЕМАТИЧЕСКИХ И ТЕХНИЧЕСКИХ НАУК. 1963, № 2

\title{
ОЩЕНКА ОБЛАСТИ РАСПОЛОЖЕНИЯ СОСТАВА ТРОЙНОГО АЗЕОТРОПА НА КОНЦЕНТРАЦИОННОМ ТРЕУГОЛЬНИКЕ
}

\author{
М. СУСАРЕВ, Л. КУДРЯВЦЕВА
}

Широко распространенное в природе явление азеотропии существенно отражается на равновесных свойствах систем раствор - пар. Отсутствие или наличие азеотропа, тип последнего и концентрационная область его расположения являются основными факторами, определяющими поведение системы в процессах перегонки и ректификации. В связи с тем, что выделение тройных азеотропов, в особенности седловых, зачастую весьма трудоемко, большое значение прнобретают работы, имеющие целью предсказание свойств тройных азеотропов на основе данных о бинарных системах, входящих в тройную.

Существующие в настоящее время методы предсказания основаны на использовании известной или предполагаемой зависимости химических потенциалов компонентов системы от состава раствора (например зависимости, характерной для регулярных растворов). Подобные методы расчета состава тройного азеотропа предложены, например, Р. Хаазе $\left[^{1}\right]$ и В. Малесинским $\left[{ }^{2},{ }^{3},{ }^{4}\right]$. Такой подход приводит к весьма конкретным результатам, оказывающимся, однако, часто ошибочными из-за значительного расхождения между предполагаемой и фактической зависимостью. Учитывая это, следует полагать, что существенное значение для достижения указанных целей должно иметь изучение свойств азеотропных систем с помощью дифференциальных соотношений, в основе.которых лежат лишь общетермодинамнческие условия равновесия и устойчивости.

Подобным соотношением является, в частности, дифференциальное уравнение кривых равных распределений между сосуществующими раствором (1) и идеальным паром (2) двух компонентов тройной системы [5].

Уравнение может быть представлено в следующей форме, удобной для практических щелей:

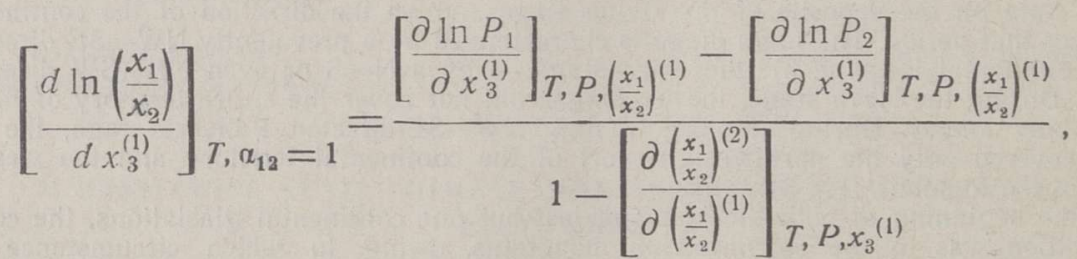

тде $P_{i}$ - парциальное давление пара $i$-го компонента;

$x_{i}^{(r)}$ - молярная доля $i$-го компонента в фазе $r$.

Нндекс $\alpha_{i k}=1$ означает, что производная берется вдоль кривой равных распределений.

На кривой равных распределений $\left(\frac{x_{i}^{(2)}}{x_{i}^{(1)}}\right)$ компонентов 1 и 2 выполняются соотно- 


$$
\frac{x_{1}^{(2)}}{x_{1}^{(1)}}=\frac{x_{2}^{(2)}}{x_{2}^{(1)}} \text { и } \quad \alpha_{12}=\frac{x_{1}^{(2)} / x_{1}^{(1)}}{x_{2}^{(2)} / x_{2}^{(1)}}=1
$$

где $\boldsymbol{\alpha}_{i k}$ - относительная летучесть. ний.

Тройной азеотроп отвечает точке пересечения трех кривых равных распределе-

Знак и величина производной (1) характеризуют направление и величину отклонения хода кривой равных распределений в какой-либо ее точке от направления, совпадающего с секущей, соединяющей рассматриваемую точку с вершиной компонента 3. Знак знаменателя правой части (1) определяется типом отклонений от законов идеальных растворов при взаимодействии компонентов 1 и 2, и его оценка никогда не вызывает затруднений. При положительных отклонениях знаменатель больше нуля, при отрицательных - меньше.

Числитель характеризует разделяющее действие компонента 3 по отношению к компонентам 1 и 2. Его знак может быть определен часто на основе знания химической природы компонентов; но обычно последнего бывает недостаточно.

Мы предлагаем способ оценки знака числителя на основе минимума исходных данных, суть которого ясна из следующих приближенных преобразований числителя:

$$
\begin{aligned}
& \left.\left.\left(\frac{\partial \ln P_{1}}{\partial x_{3}^{(1)}}\right)_{T, P,\left(\frac{x_{1}}{x_{2}}\right.}\right)^{(1)}-\left(\frac{\partial \ln P_{2}}{\partial x_{3}^{(1)}}\right)_{P, T,\left(\frac{x_{1}}{x_{2}}\right.}\right)^{(1)} \simeq\left(\frac{\partial \ln P_{1}}{\partial x_{3}^{(1)}}\right)_{T, P,\left(\frac{x_{1}}{x_{2}}\right)^{(1)}=\infty}- \\
& -\left(\frac{\partial \ln P_{2}}{\partial x_{3}^{(1)}}\right)_{P, T,\left(\frac{x_{1}}{x_{2}}\right)^{(1)}=0} \sim \frac{\Delta P_{1}^{13}}{P_{1}^{0} \cdot \Delta x_{3}^{13}}-\frac{\Delta P_{2}^{23}}{P_{2}^{0} \cdot \Delta x_{3}^{23}}=\frac{P_{1}^{13}-P_{1}^{0}}{P_{1}^{0} \cdot x_{3}^{13}}-\frac{P_{2}^{23}-P_{2}^{0}}{P_{2}^{0} \cdot x_{3}^{23}},
\end{aligned}
$$

где $P_{1}^{13}$ и $P_{2}^{23}$ - парциальное давление пара компонентов 1 и 2 в азеотропах $1-3$ и $2-3$ соответственно,

$P_{1}^{0}$ и $P_{2}^{0}$ - давление пара чистых компонентов 1 и 2 при температуре кипения азеотропа $1-3$ и $2-3$ соответственно,

$x_{3}^{13}$ и $x_{3}^{23}$ - молярная доля третьего компонента в азеотропах $1-3$ и $2-3$.

Из (3) видно, что в основе преобразований лежит предположение о возможности оценки разделяющего действия компонента 3 (на кривой равных распределений компонентов 1 и 2) путем сравнения относительных изменений величин $P_{1}$ и $P_{2}$ при изменении состава бинарных систем $1-3$ и $2-3$, т. е. на основе сопоставления взаимодействия компонента 3 с компонентами 1 и 2, взятыми порознь.

Оценивая знак числителя (1) по соотношению (3) и учитывая знак знаменателя, можно определить, по какую сторону от каждой из секущих, соединяющих составы бинарных азеотропов с противолежащими вершинами треугольника, должен располагаться тройной азеотроп.

При таком способе оценки выявляемая область расположения тройного азеотропа обычно занимает еще довольно значительную часть площади треугольника составов. Ей, в частности, всегда принадлежит одна из вершин треугольника. Между тем ясно, что, если составы бннарных азеотропов располагаются вдали от вершин, то тройной азеотроп не будет располагаться вблизи какой-либо из них. Его расположение должно быть как-то связано с той областью треугольника, в которой располагаются бинарные азеотропы. Несомненно также, что характер этой связи должен зависеть и от типа бинарных азеотропов, как это имело место и ранее. В связи с этими соображениями мы предлагаем следующее правило, налагающее дополнительные ограничения на область расположения тройного азеотропа, выявляемую способом, изложенным выше.

При наличии в системе тройного азеотропа состав последнего располагается внутри (вне) треугольника, вершинам которого отвечают составы бинарных азеотропов, если тип последних одинаков (различен).

Строгое обоснование этого положения, по-видимому, может быть дано на основе анализа дифференциального уравнения кривых равного содержания компонента в 


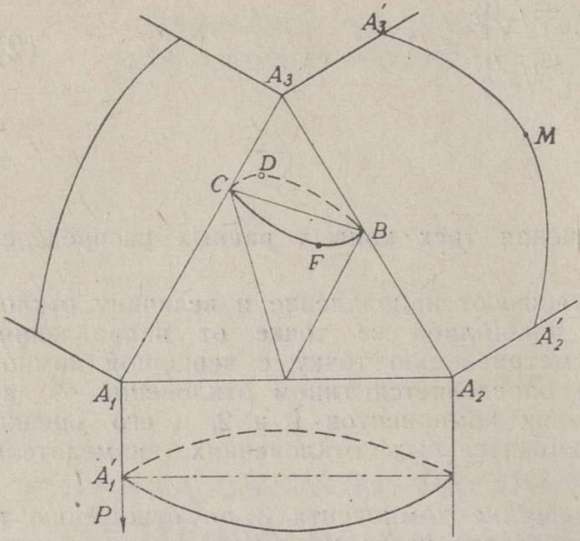

этанол (3)

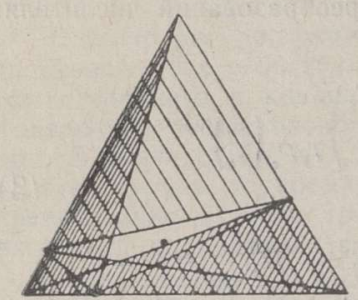

хлороформ (1)

сероуглерод

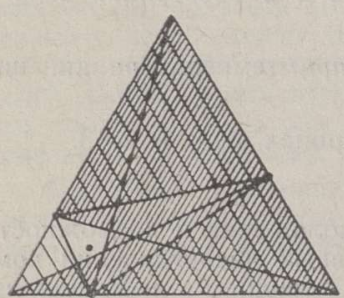

метилформиат

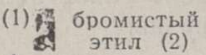

4

(1) нитробензол

(3)

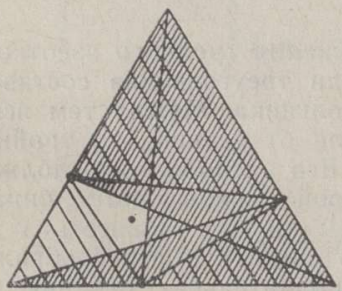

бензиловый спирт (1) 7 гексан

(2)

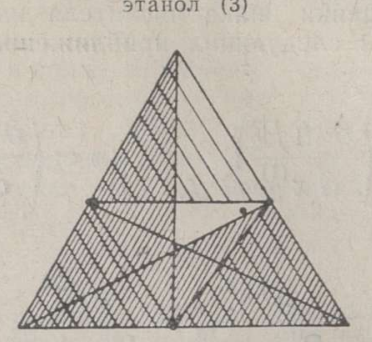

сосуществующих фазах $\left(x_{i}^{(1)}=x_{i}^{(2)}\right)$, которым принадлежат составы тройного и двух бинарных азеотропов, образуемых компонентом $i$. Здесь мы ограничимся качественным обоснованием, исходя из общепринятых представлений о непрерывном характере изменения свойств систем рассматриваемого типа при изменении состава.

На рис. 1 изображена тройная азеотропная система. Зависимость давления пара бинарных растворов от состава представлена на участках плоскости, примыкаю-

Рис. 1. 
щих к сторонам концентрационного треугольника. В бинарных системах $A_{1} A_{3}$ и $A_{2} A_{3}$ давление пара имеет максимум. Для системы. $A_{1} A_{2}$ представлено три возможных варианта, в том числе случай, когда давление является линейной функцией состава. Поверхность давления пара тройной системы будем рассматривать как поверхность, образованную путем пространственного перемещения кривой $A_{2}^{\prime} A_{3}^{\prime}$ (образующей), конец которой $A_{3}^{\prime}$ закреплен, а конец $A_{2}^{\prime}$ движется по кривой $A_{1}^{\prime} A_{2}^{\prime}$ (направляющей). Предполагается, что при этом движении (на пути от $A_{2} A_{3}$ к $A_{1} A_{3}$ ) образующая изменяет свой вид от одной предельной формы к другой непрерывным образом.

Если направляющая $A_{1}^{\prime} A_{2}^{\prime}$ является прямой, то точка $M$ на образующей опишет при своем движении путь, представленный на треугольнике концентраций линией $B C$, соединяющей составы бинарных азеотропов, близкой к прямой. Если направляющая $A_{1}^{\prime} A_{2}^{\prime}$ вогнута по отношению к $A_{1} A_{2}$, точки, отвечающие максимуму $p$ на перемещающейся образующей, опишут кривую $B D C$, располагающуюся между прямой $B C$ и вершиной $A_{3}$. В противоположном случае (положительные отклонения в системе $A_{1} A_{2}$ ) мы получим кривую $B F C$, располагающуюся по другую, чем вершина $A_{3}$, сторону от прямой $B C$. Сопоставление заключений, которые можно сделать при рассмотрении трех вариантов образования поверхности путем перемещения кривых $A_{2}^{\prime} A_{3}^{\prime}, A_{1}^{\prime} A_{2}^{\prime}$ и $A_{1}^{\prime} A_{3}^{\prime}$ приводит к положению, сформулированному выше. Рассмотрение систем, в ко-

циклогексан (3)

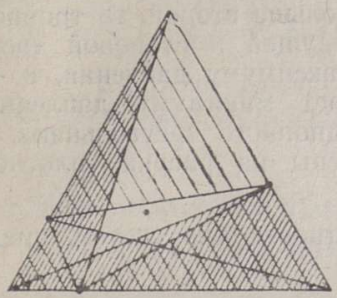

ацетон (1) 10

гексан (3)

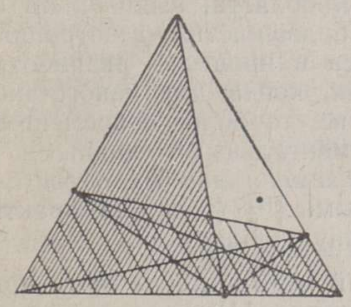

Min

13

уксусная кислота

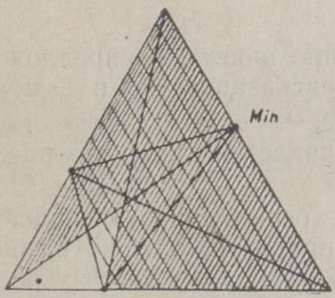

H-гептан (1) метанол (2) н-пропанол (1)

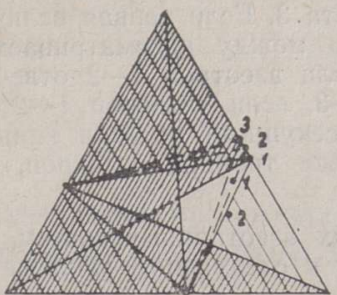

11 вода (2) метанол (3)

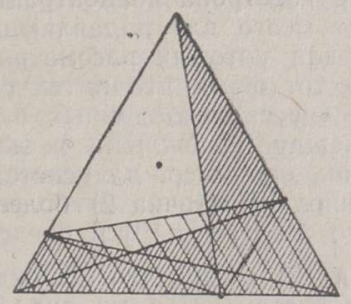

Min

хлоро- ацетон (1)

14

хлоро- уксусная

кислота (1)

15

хлороформ

(3)

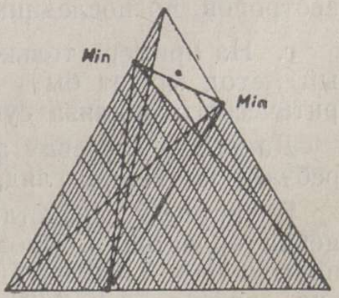

Min

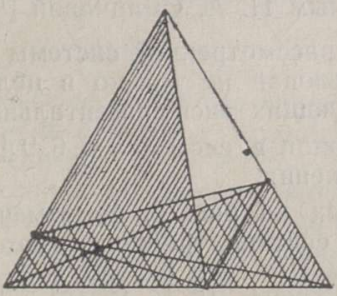

o-крезол воді (3)

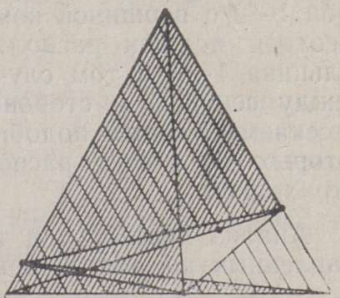

12

бензол (2)

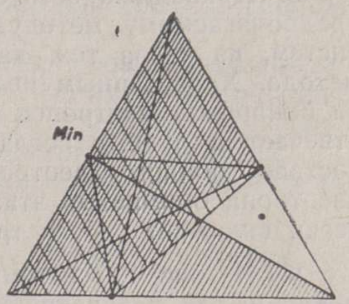

H-октан (2) 
торых наличие тройного азеотропа не связано с наличием трех бинарных азеотропов, может быть также проведено на принципах, изложенных выше.

Обсуждение предложенного метода ощенки области расположения состава тройного азеотропа на концентрационном треугольнике мы свяжем с иллюстративным материалом, представленным на рис. 2.

Компоненты каждой из 18 систем, представленных на рисунке, образуют тройной и три бинарных азеотропа. Тройные азеотропы систем с 1 по 12 отвечают максимальной точке поверхности давления, остальных шести систем - седловой точке (отрицательным бинарным азеотропам отвечает обозначение Min).

По данным о составах бинарных азеотропов был выявлен треугольник, вершины которого отвечают этим составам. Та область, в которой, согласно предложенному правилу, тройной азеотроп не должен располагаться, покрыта редкой штриховкой.

С помощью данных о-составах бинарных азеотропов и о величинах давления пара чистых компонентов при температурах кипения бинарных азеотропов были определены относительные понижения давления пара компонентов $\left(\frac{P_{i}^{i k}-P_{i}^{0}}{P_{i}^{0}}\right)$ при переходе последних из чистого состояния в бинарные азеотропные растворы. Сопоставление величин $P_{1}^{13}-P_{1}^{0} \quad P_{2}^{23}-P_{2}^{0}$

$\frac{1}{P_{1}^{0} \cdot x_{3}^{13}}$ и $\frac{P_{2}}{P_{2}^{0} \cdot x_{3}^{23}}$ и учет типа бинарного азеотропа $1-2$ дает возможность определить.

расположение состава тройного азеотропа относительно секущей, соединяющей азеотроп 1-2 с вершиной компонента 3. Если первая величина больше второй, то тройной азеотроп должен располагаться между рассматриваемой секущей и стороной треугольника 1-3 в том случае, если азеотроп $1-2$ отвечает максимуму давления, и между секущей и стороной 2-3, если азеотроп $1-2$ отвечает минимуму давления. Отсекаемые тремя подобными секущими области концентрационного треугольника, в которых не может располагаться тройной азеотроп, отмечены на рнсунке плотной штриховкой.

Анализ данных для тройных азеотропных систем, в частности для систем, представленных на рис. 2, приводит к следующим заключениям:

а. Составы тройных азеотропов систем 1,2, 3 и 13, экспериментально исследованных одним из авторов $[6,7,8,9,10]$, все без исключения располагаются в тех областях концентрационного треугольника, которые были выявлены с помощью обсуждаемого метода;

б. Принадлежность состава азеотропа концентрационной области, выявленной по предполагаемому методу, имеет место для подавляющего большинства азеотропных систем, из числа тех, данные для которых рассматривались в процессе разработки метода. Характерным является тот факт, что в тех случаях, когда для какого-либо из бинарных азеотропов имеется несколько данных о составе, то более современным отвечает и лучшее согласие между оценочными и экспериментальными данными о составе тройного азеотропа. Так, например, для системы 8 замена данных о составе азеотропа бромистый этил-метанол $\left[{ }^{11}\right]$ (точка 2) более новыми $\left[{ }^{12}\right]$ (точка 1) практически включает состав тройного азеотропа [11] в предсказанную область;

в. Из рис. 2 (системы 11 и 12) видно, что обсуждаемый метод может быть применен и для расслаивающихся систем. Данные рис. 2 (система 11) очень наглядно показывают также, как обновление экспериментальных данных изменяет имевшее местонесоответствие на полное соответствие между предсказанной областью расположения тройного азеотропа и его составом, выявленным экспериментально. Точкой 3 , по данным Хеннота $\left[{ }^{13}\right]$, обозначен состав бинарного азеотропа, точками 2 - состав бинарного и тройного азеотропов, по данным справочника Хорсли, точками 1 - составь азеотропов, по последним данным Н. А. Смирновой $\left[{ }^{14}, 15\right]$

г. На примере только что рассмотренной системы нетрудно видеть, что предложенный метод может быть использован не только в целях предсказания, но и в целяз критического анализа существующих экспериментальных данных.

Данные о составах азеотропов в системах $5,6,9\left[{ }^{11},{ }^{12}\right]$, представленных на рис. 2. требуют, на наш взгляд, уточнения.

В системах, представленных на рис. 2, не отмеченных особо при обсуждении, оценка области расположения состава тройного азеотропа производилась по данным справочников Хорсли [11] и Лека [12] (для систем $4,7,10,12,14$ ) и по данным польских авторов $[16,17,18,19]$ (для систем $15,16,17,18$ ). Для систем, не исследованных нами, данные о давлении пара чистых компонентов при.температурах кипения соответствующих азеотропов были получены путем интерполяции данных таблик Стэлла [20]. 


\title{
Л И Т Е Р А Т У Р А
}

1. R. H a a s e, Z. phys. chemie, $195,362,1950$.

2. В. М а ле с и н с ки й, Бюлл. Польск. АН, отд. III, 3, 599, 1955.

3. В. М а л е с и н с к й, Бюлл. Польск. АН, отд. III, 5, 295, 1956.

4. В. М а л е с и н с ки й, Бюлл. Польск. АН, отд. III, 10, 693, 1956.

5. М. П. С у с а р в, Ж.Ф.Х. в печати.

6. Л. С. Кудр явце в а и М. П. С у с а е в, Ж.П.Х., № 6, 1963.

7. Л. С. Куд р я в е в а и М. П. С у с а рев, Ж.П.Х., № 7, 1963.

8. Л. С. К уд р я вце в а и М. П. С у с а р е в, Ж.П.Х., № 8, 1963.

9. Л. С. К удря в ц в а и М. П. С у с а ре в, Ж.П.Х., № 9, 1963.

10. А. В. С торонкин, А. Т. Морачевский, Л. С. Кудрявцева, Ж.Ф.Х. 31, 395, 1957.

11. Л. Х о р сли, Таблицы азеотропных смесей. ИЛ, 1951.

12. M. L e c a t, Tables azeotropiques I, Uccle, Bruxelles, 1949.

13. Th. H a n not e, Bull. Soc. Chim. Belg. 35, 85, 1926.

14. Н. А. С ми нова, А. Г. Морачевский и А. В. Стөронкин, Вестник ЛГУ, 22, 70, 1959.

15. Н. А. С м и н о в а, А. Г. Мор а че в ки й, ЖФХ, 34, 2546, 1960.

16. K. Z i eborak and D. Wy rz y k w ska-Stanki ew i ez, Бюлл. Польск. AH, сер. хим., 6, 6, 377, 1958.

17. К. З ем борак и И. 3 е м бор ак, Бюлл. Польск. АН, отд. III, 2, 6, 291, 1954 .

18. 3. К у р ты к а, Бюлл. Польск. АН, отд. III, 4, 7, 47, 1956.

19. А. Орсаг, Я. Л еляковская и М. Белдович, Бюлл. Польск. АН, сер. хим., 6, 7, 419, 1958.

20. Д. Р. С тэлл, Таблицы давления паров индив. веществ. ИЛ, 1949.
Ннститут химии
Академии наук Эстонской ССР
Поступила в редакцию
5. III 1963

\section{KOLMEKOMPONENDILISE ASEOTROOBI KOOSTISE ASUKOHA PIIRKONNA MÄĂRAMINE KONTSENTRATSIOONI KOLMNURGAL}

\author{
M. Sussarev, L. Kudrjavtseva
}

\section{Resümee}

Artiklis konkretiseeritakse varem esitatud reegli (5) praktilise kasutamisega seoses olevaid küsimusi. Esitatakse uus reegel, mis täiendavalt piirab aseotroobi koostise asukoha kontsentratsioonipiirkonda.

Kolmekomponendilise aseotroobi olemasolu puhul asub viimase koostis kontsentratsioonikolmnurgal sisemise kolmnurga sees, kui selle tippudele vastavad ühetüübiliste binaarsete aseotroopide koostised. Kolmikaseotroobi koostis asub aga väljaspool sisemist. kolmnurka, kui selle tippudele vastavad eritüübiliste binaarsete aseotroopide koostised.

Kolmikaseotroobi koostise kontsentratsioonipiirkonna määramise meetod illustreeritakse kaheksateistkümne kolmekomponendilise süsteemiga.
Eesti NSV Teaduste Akadeemia Keemia Instituut
Saabus toimetusse
5. III 1963

\section{ESTIMATION OF THE REGION OF PLACEMENT OF THE COMPOSITION OF A TRIPLE AZEOTROPE ON A CONCENTRATIONAL TRIANGLE}

\author{
M. Susarev, L. Kudryavtseva
}

\section{Summary}

In the article questions are elucidated which are connected with the application of at formerly proposed rule (5) permitting to define the concentrational region of the placement of the composition of a triple azeotrope. A new rule is proposed imposing further limitations on the region of the placement of the composition of the azeotrope:

in the presence, in the system of a triple azeotrope, the composition of which is placed within (without) the triangle, to whose apexes correspond to the compositions of the binary azeotropes if the type of the latter is similar (different).

The authors present an illustration of the proposed method on the example of eighteen triple azeotropic systems.

$$
\begin{gathered}
\text { Academy of Sciences of the Estonian S.S.R., } \\
\text { Institute of Chemistry }
\end{gathered}
$$

Received March 3rd, 1963 